\title{
Nube híbrida nacional: soberana, libre, interoperable y con desarrollo local
}

\author{
Leandro Monk ${ }^{1}$, Fernando Schapachnik ${ }^{2}$, Sebastián Uchitel ${ }^{3}$, Pablo \\ Vanninii $^{4}$, Mariano Zukerfeld ${ }^{5}$, Andrea Díaz ${ }^{6}$, Julián Dunayevich ${ }^{7}$, Juan \\ Lagostena $^{8}$ y Nicolás Passerini ${ }^{9}$
}

Recibido: 20/05/2021; Aceptado: 25/05/2021

Cómo citar: Monk, M.; Schapachnik, F.; Uchitel, S.; Vannini, P.; Zukerfeld, M.; Díaz, D.; Dunayevich, J.; Lagostena, J. y Passerini, N. (2021) Nube híbrida nacional: soberana, libre, interoperable y con desarrollo local. Revista Hipertextos, 9 (15), 201-205. DOI: https://doi.org/10.24215/23143924e036

Recientemente la Secretaría de Innovación Pública ha convocado a un procedimiento de consulta para la concreción de una "Nube Híbrida de Gobierno". El objetivo de la Consulta es recibir aportes, ideas, experiencias y recomendaciones para la definición de criterios tecnológicos que permitan la construcción de la misma.

En este marco, quienes firmamos la presente, actores de diversas organizaciones del quehacer tecnológico nacional, mujeres y hombres con décadas de experiencia profesional en tecnologías informáticas y su relación con el desarrollo nacional, apoyamos la construcción de una "Nube híbrida de Gobierno", saludamos el llamado a Consulta y acordamos en una serie de principios que creemos fundamentales para su desarrollo y el aseguramiento de la soberanía tecnológica. Para comenzar señalamos que consideramos conveniente utilizar el término "nube híbrida nacional", más apropiado en nuestro idioma para el concepto de bybrid gov cloud que se encuentra en la bibliografía sobre el tema.

Este concepto nos permite pensar la importancia de la independencia tecnológica para tener un control real sobre las tecnologías y por lo tanto sobre las capacidades del Estado. Implica que el Estado debe tener control legal, intelectual y operativo de la infraestructura informática y el software (IIS) que es crítica para la gestión de polí-ticas públicas. Sin soberanía tecnológica, el

\footnotetext{
${ }^{1}$ gcoop - FACTTIC

2 Fundación Sadosky \& ICC UBA-CONICET

${ }^{3}$ Director Instituto UBA/CONICET de Ciencias de la Computación

${ }^{4}$ gcoop - UNPAZ

${ }^{5}$ Conicet - e-TCS/Centro CTS/Umai

${ }^{6}$ Lic en Ciencias de la Computación, UBA. Esp en Gestión de la Tecnología y la Innovación, UNSAM

7 Lic. en Ciencias de la Computación

8 Ing. en Informática, docente universitario

${ }^{9}$ Ingeniero en Sistemas de Información, docente universitario. Director de Seguridad de la Información en AFIP
} 
país se desprende de grados de libertad para adoptar las políticas tecnológicas que le resulten convenientes, restricción que a su vez impacta en cómo despliega el aspecto tecnológico de sus políticas, que es cada vez de mayor preeminencia.

Consideramos que la nube híbrida nacional puede y debe ser desarrollada en base a tecnologías libres, interoperables, que aseguren independencia al Estado de cualquier proveedor de software o infraestructura de cómputo.

Existen en el país personas e instituciones públicas y privadas con los conocimientos que permiten desarrollar la nube híbrida nacional generando trabajo y desarrollo local. En base a tecnologías libres y con personal local, el Estado puede asegurar el cumplimiento tanto de las normativas como de los más altos estándares en cuanto a la seguridad de la información y el resguardo de los datos personales de la ciudadanía.

Como señala la convocatoria la nube pública debe evitarse el vendor lock-in, es decir, las ataduras a uno o varios proveedores, asegurando mediante estándares abiertos la interoperabilidad con otras infraestructuras públicas o privadas.

Las siguientes secciones discuten distintos aspectos de las posibilidades que consideramos que se abren si la nube híbrida nacional se desarrolla bajo los principios de soberanía, software libre e interoperabilidad.

\section{Visión}

Conceptualizamos a la nube híbrida de Gobierno en base a algunos principios ordenadores y de organización.

Principios ordenadores de todo el proyecto:

- Soberanía tecnológica.

- Software libre.

- Interoperabilidad.

Principios de organización:

- Contrataciones bajo legislación argentina y en moneda nacional. Los servicios de la nube híbrida deben poder ser contratables mediante contratos enmarcados en su totalidad en el ordenamiento legal argentino, y pagables en moneda nacional.

- Convivencia de múltiples proveedores de manera transparente. Los usuarios de la nube deben contar con un conjunto de servicios que sean provistos de manera transparente por multiplicidad de proveedores. El usuario debe poder migrar sus servicios de uno a otro de manera transparente.

- Existencia de infraestructura propia. Entre esos múltiples proveedores es indispensable que la propia Arsat sea uno de ellos y que brinde servicios completamente alojados en su propio datacenter de manera de asegurar los máximos 
niveles de soberanía y observancia a las leyes nacionales.

- Inclusión de otros organismos, entidades y empresas. Otros organismos públicos y entidades (cooperativas, universidades, empresas, etc.) deben poder sumar su infraestructura a la nube híbrida de manera tal de poder gestionar sus recursos propios y los arrendados a otros actores de la nube de manera integrada, pero a su vez de poner ofrecer su capacidad de cómputo excedente para que pueda ser utilizada por otras actores del ecosistema híbrido.

- Transparencia respecto a los niveles de calidad de servicio. Los distintos actores participantes en la nube brindarán servicios con diferentes parámetros de calidad.

- Tendrán distintos precios.

- Garantizarán o no que sus servicios se prestan desde el país (soberanía)

- Garantizarán distintos parámetros de red (ancho de banda, latencia, redundancia, etc.).

- Garantizarán distinta calidad de equipamiento.

A la hora de elegir cualquiera de los proveedores dentro de la red debería ser claro para el usuario cuáles son las características que le están siendo ofrecidas en todas estas dimensiones. Será función de Arsat certificar que los parámetros efectivamente ofrecidos coincidan con los declarados.

\section{Soberanía tecnológica}

Es importante destacar que hoy en día la implementación de casi la totalidad de las políticas públicas requiere algún soporte informático. Desde el análisis de grandes datos de salud en el contexto de la pandemia de covid, hasta de las políticas de desarrollo social como la AUH. Este nivel de informatización de la gestión del Estado sólo va a crecer en los próximos años, a un ritmo cada vez más acelerado.

La tecnología de nube aparece en el centro de cualquier implementación tecnológica de hoy en día. Es por eso que el Estado debe asegurarse su control legal, intelectual y operativo. Una nube basada en tecnologías libres permite que el Estado conozca todos los detalles de la tecnología que emplea y le da la posibilidad de tomar sus propias decisiones tecnológicas.

\section{Despliegue federal y multi actor}

Para el país, sumar la mayor cantidad de recursos de una manera inteligente para su disponibilización y uso es muy importante. Para ello es mandatorio que pensemos esta política pública como la posibilidad de integrar ordenadamente toda o la mayoría de la infraestructura de los grandes y medianos organismos públicos nacionales, provinciales y municipales. Estos que ya cuentan con infraestructura propia y normativa dura, en materia de tratamiento de datos, por ejemplo. Podrían sumar sus equipos actuales o de inversiones futuras en un esquema donde, por ser nube híbrida, en función de esas necesidades tener la posibilidad de migrar servicios ante determinado contexto y elegir el proveedor que mejor se ajuste a esas necesidades.

La nube pública híbrida es además un espacio donde cada actor puede maximizar sus recursos digitales: Gobiernos Provinciales y Municipales, los centros de investigación y universidades, las empresas públicas y bancos públicos, las empresas de desarrollo de software, las empresas 
nacionales de infraestructura, los sindicatos, organizaciones del mundo del trabajo y sus trabajadores.

\section{Seguridad de la información}

En un modelo de computación en en la nube, los usuarios del servicio entregan sus datos y delegan diferentes formas de cómputo en el proveedor de la plataforma, que se responsabiliza por garantizar la confidencialidad, integridad y disponibilidad de los datos y computaciones. El Estado, al brindar o promover la existencia de un servicio de Nube Híbrida, tiene la obligación irrenunciable de velar porque el servicio provisto cumpla con estas cualidades. Estas responsabilidades sólo pueden cumplimentarse en tanto el Estado reserve para sí la capacidad de controlar cada uno de los componentes que intervengan para la provisión de los servicios.

\section{Generación de empleo}

La nube híbrida nacional puede tener impactos virtuosos en la cantidad y calidad del empleo argentino al menos mediante dos mecanismos. El primero se asocia a la creación directa de empleos y consiste en reemplazar parte del masivo trabajo que realizan las plataformas extranjeras y privadas; plataformas que, en muchos casos, no respetan las condiciones de trabajo estipuladas por los marcos legales nacionales ni ofrecen remuneraciones justas.

El segundo mecanismo refiere a que, de manera indirecta, la nube híbrida de gobierno puede impulsar el crecimiento de las firmas del sector SSI argentino y así incrementar la cantidad y calidad del empleo en el sector. De hecho, pese a su sostenido crecimiento y su capacidad para generar trabajo y agregar valor, el sector se encuentra aún poco integrado con el Estado. La nube híbrida es, por ello, una excelente oportunidad para demandar desarrollos y servicios del sector SSI que a su vez, generen más empleo local e, incluso, para hacerlo priorizando a las empresas que garanticen condiciones de trabajo y remuneraciones justas para para sus trabajadores y trabajadoras.

\section{Ahorro de divisas}

Una de las ventajas del modelo de nube híbrida propuesto es que se reduce sustancialmente la erogación de divisas o incluso en algunos casos se puede hasta evitar. Esto encuentra su fundamento en el hecho de que:

- Si lo hacemos con herramientas open source o de código abierto, muchas veces se puede evitar tener que recurrir a soluciones pagas provistas por proveedores extranjeros bajo la modalidad de licencias.

- Dado que el hardware es ofrecido por ARSAT, y por consiguiente, adquirido en gran escala, el costo es significativamente más bajo.

- Dado que el hardware se encuentra virtualizado a través de tecnologías como OpenStack y Kubernetes, entre otras, es posible no depender de un soporte físico y, a su vez, 
consumir recursos sólo cuando se necesita, en lugar de hacerlo todo el tiempo. Esto a fin de cuentas se traduce en una optimización en la administración de dichos recursos.

- Un modelo de software como servicio (SaaS) permite generar sobre la base de una plataforma común una cartera de herramientas articuladas entre sí que pueden utilizarse para diversos fines en toda la organización: Recursos Humanos, Presupuesto, Patrimonio, etc. Así, se evita recurrir a soluciones externas y se optimiza el desarrollo de sistemas brindando herramientas que pueden utilizarse en todo el Estado Nacional.

- Generalmente, al adquirir software licenciado, resulta casi indispensable sumar un ítem de capacitación al presupuesto para poder hacer uso de las soluciones y éste suele ser bastante alto. Por el contrario, en un modelo como éste, el desarrollo de herramientas comunes para diversos grupos de trabajo, habilita la generación de comunidades donde el aprendizaje en el uso de las herramientas puede darse de una manera más transversal y colaborativa, atravesado por un marco de conocimiento global e integral.

- Lo anterior a su vez permite contar con consultores locales y no depender de servicios de consultoría externa que se facturan en dólares.

\section{Elaboración del documento}

Las siguientes personas participaron de la elaboración del documento a título individual.

- Leandro Monk, gcoop - FACTTIC

- Fernando Schapachnik, Fundación Sadosky \& ICC UBA-CONICET

- Sebastian Uchitel, Director Instituto UBA/CONICET de Ciencias de la Computación.

- Pablo Vannini, gcoop - UNPAZ

- Mariano Zukerfeld- Conicet - e-TCS/Centro CTS/Umai

- Andrea Díaz, Lic en Ciencias de la Computación, UBA. Esp en Gestión de la Tecnología y la Innovación, UNSAM.

- Julián Dunayevich. Lic. en Ciencias de la Computación.

- Juan Lagostena, Ing. en Informática, docente universitario.

- Nicolás Passerini, Ingeniero en Sistemas de Información, docente universitario. Director de Seguridad de la Información en AFIP

El documento completo puede leerse en: http://www.cvi.com.ar/nube_hibrida_nacional.pdf 